\title{
Posterior Repair with Perforated Porcine Dermal Graft
}

\author{
G. Bernard Taylor, Robert D. Moore, John R. Miklos, T. Fleming Mattox
}

Department of Obstetrics and Gynecology (GBT), Carolinas Medical Center, Charlotte, North Carolina, Atlanta Urogynecology Associates (RDM, JRM), Atlanta, Georgia, Greenville Hospital System (TFM), Center for Women's Medicine, Greenville, South Carolina, USA

\begin{abstract}
Objective: To compare postoperative vaginal incision separation and healing in patients undergoing posterior repair with perforated porcine dermal grafts with those that received grafts without perforations. Secondarily, the tensile properties of the perforated and non-perforated grafts were measured and compared.

Materials and Methods: This was a non-randomized retrospective cohort analysis of women with stage II or greater rectoceles who underwent posterior repair with perforated and non-perforated porcine dermal grafts (Pelvicol ${ }^{\mathrm{TM}} \mathrm{CR}$ Bard Covington, GA USA). The incidence of postoperative vaginal incision separation (dehiscence) was compared. A secondary analysis to assess graft tensile strength, suture pull out strength, and flexibility after perforation was performed using standard test method TM 0133 and ASTM bending and resistance protocols.

Results: Seventeen percent of patients (21/127) who received grafts without perforations developed vaginal incision dehiscence compared to $7 \%(5 / 71)$ of patients who received perforated grafts $(\mathrm{p}=0.078)$. Four patients with vaginal incision dehiscence with non-perforated grafts required surgical revision to facilitate healing. Neither tensile strength or suture pull out strength were significantly different between perforated and non-perforated grafts $(p=0.81, p=0.29$, respectively). There was no difference in the flexibility of the two grafts $(\mathrm{p}=0.20)$.

Conclusion: Perforated porcine dermal grafts retain their tensile properties and are associated with fewer vaginal incision dehiscences.
\end{abstract}

Key words: rectal prolapse; female; surgery; graft; porcine

Int Braz J Urol. 2008; 34: 84-90

\section{INTRODUCTION}

The use of interpositional grafts to reinforce weakened or absent endopelvic fascia and augment pelvic floor repairs has become common practice in recent years. Both synthetic and biologic materials have been used in nearly every facet of reconstructive pelvic surgery, including pubovaginal slings and anterior and posterior repairs, as well as abdominal sacral colpopexy (1-6). The purpose of using grafts is to provide a tissue scaffold for ingrowth to occur and improve tissue strength, thereby improving the longevity of the surgical repairs. Posterior repairs performed by using patient native tissue via colporrhaphy or site specific defect repair have failure rates of $14-39 \%$ (7). Early studies have shown it to be 
comparable to other graft materials with a greater than 90\% success rate in corrective procedures for pelvic organ prolapse $(3,5)$. While rare, complications related to vaginal incision healing have been reported $(3,5$, 8). Graul et al. reported eight incisional separations or graft expulsions in their initial series of 141 repairs augmented with porcine dermal grafts. Moore et al also found that $20 \%$ of their patients experienced graft related complications primarily related to early incision healing. More recently, cases of porcine dermal graft suburethral sling encapsulation and tissue reactions resulting in variable outcomes have also been reported.

The porcine dermal graft is an acellular collagen matrix that is biocompatible and theoretically provides a scaffold for native tissue ingrowth and remodeling over a period of weeks to months following implantation. It is essentially nonporous, presenting only surface pores (from hair follicles) of inconsistent and uncertain size. While the majority of wounds heal well, because of the nonporous nature of the porcine dermal graft there is evidence that tissue ingrowth and remodeling may not occur in all cases.

In our initial experience with porcine dermal grafts used in patients to augment posterior repairs for rectocele, we noted a number of patients with wound separations (dehiscences) during the postoperative period. Many of these patients were felt to have wound hematomas and/or seromas associated with the porcine dermal graft wound dehiscences. As a result we began perforating the grafts to facilitate drainage across the graft during the early postoperative period. This is a report of our initial experience using porcine dermal graft perforation in posterior repair.

\section{MATERIALS AND METHODS}

This study is a retrospective cohort analysis of all patients who had undergone posterior repair with graft augmentation for rectocele using perforated porcine dermal grafts.

The patients underwent surgery between January 1, 2003 and July 30, 2003 at Northside Hospital, Atlanta, Georgia. A control group of patients who had undergone a posterior repair with nonperforated porcine grafts served as controls.
Demographic data and physical examinations were included on all patients. Postoperative healing, specifically incision dehiscence occurring within the immediate 4-6 week postoperative period, was compared. The research protocol was reviewed and approved by the Institutional Review Board at Northside Hospital in Atlanta, Georgia.

Secondarily, to assess graft integrity after perforation tensile strength, suture pull out strength and flexibility tests were performed on the porcine grafts. Tensile strength was performed with an Instron $^{\mathrm{TM}}$ tensile testing machine. The graft samples were cut to 2 x 3.5 centimeters $(\mathrm{cm})$ pieces and loaded on the machine with a separation distance of $2 \mathrm{~cm}$ and at a crosshead speed of 0.1969 inches/minute and 100 pounds (lbs) load cell. Suture pull out strength was assessed by attaching a 3-0 PDS suture 3 millimeters $(\mathrm{mm})$ from the edge of the graft, and tested as described. The grafts were stressed to failure. To test stiffness and flexibility, we used the American Society of Testing Materials standard test method 6125-97 for bending and resistance of paper and paperboard. A Gurley ${ }^{\mathrm{TM}}$ bending resistance stiffness tester set at $0.5 \mathrm{Lbs}$ at $2.0 \mathrm{~cm}$ was loaded with $0.5 \mathrm{x}$ $1.0 \mathrm{~cm}$ pieces of graft. The tests were performed on random lots of 10 each perforated and non-perforated porcine grafts.

All patients had clinical stage II or greater (ICS Pelvic Organ Prolapse Quantitative system point $\mathrm{Bp}=0$ or greater) rectoceles and elected to undergo surgical repair. The patients were taken to the operating room and, following adequate anesthesia application, were placed in the dorsolithotomy position. Following sterile preparation and draping, posterior repair was performed through an inverted-T incision extending from the perineum to the apex of the rectocele in the midline. The vaginal mucosa was sharply dissected bilaterally to expose the fascia of the levator ani muscles and superiorly to expose the intact rectovaginal/pubocervical fascia. The patients then underwent site-specific defect repair of rectocele using interrupted 2-0 Vicryl (Ethicon, Summerville, New Jersey) suture. The decision to use the porcine dermal graft was made intraoperatively based on the subjective strength of the site-specific repair and the quality of the patient's tissues. Risk factors for 
rectocele repair failure including prior rectocele repair, obesity, chronic cough or pulmonary disease and advanced prolapse were also considered in determining whether or not to use a graft.

The porcine dermal graft (Pelvicol ${ }^{\mathrm{TM}}$, C.R. Bard, Covington, GA) was used for all graft augmented repairs. A piece of $6 \times 8 \mathrm{~cm}$ porcine dermal graft was trimmed to fit the dimension of the previously dissected posterior repair extending from the superior rectovaginal/pubocervical fascia to the perineal body and levator ani fascia bilaterally. The grafts were attached to the rectovaginal/pubocervical fascia superiorly, the lavator ani fascia laterally, and the perineal body distally using interrupted 2-0 Ethibond (Ethicon, Summerville, New Jersey) suture. The vaginal epithelium was closed in a running nonlock fashion with a 2-0 Vicryl (Ethicon, Summerville, New Jersey) suture. Graft perforation was performed after removal of the porcine dermal graft from the sterile packages using a $3 \mathrm{~mm}$ key punch. A template was used to standardize the perforations to make a series of rows in $1 \mathrm{~cm}$ increments (Figures-1). All perforated grafts were placed in the same manner without alteration in technique as previously described. All of the patients received preoperative antibiotic prophylaxis using a dose of 1 gram of Cefazolin intravenously. Postoperative antibiotics were not routinely given.

Patients were initially examined four weeks postoperatively and every 2 to 4 weeks thereafter as

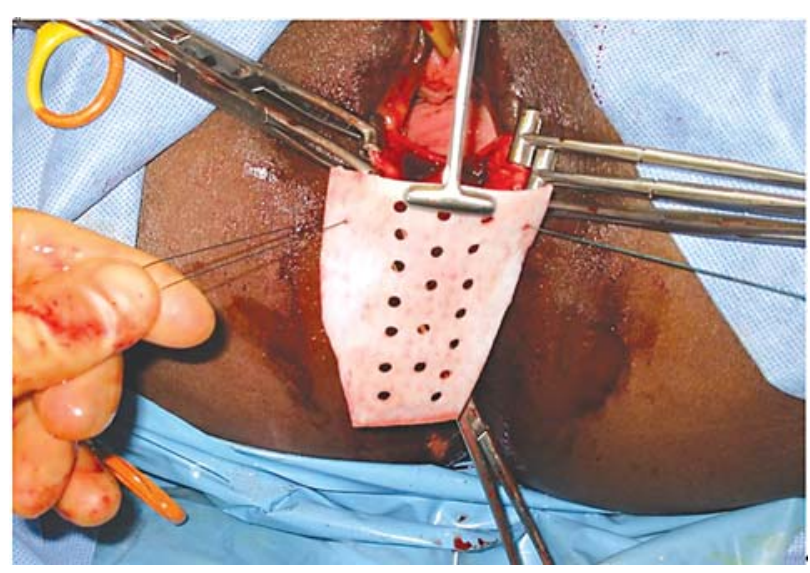

Figure 1 - Perforated Pelvicol graft. decided by the examining physician. Data regarding graft exposure, dehiscence, and expulsion was retrieved from the patient's charts. Incision dehiscence or graft exposure was defined as the ability to visually see graft material through the suture line during the initial postoperative period of 4 weeks.

The laboratory data was analyzed using the Student t test. Categorical data analysis was performed using Fischer's exact test (SAS computer software, SAS Institute, Cary, North Carolina).

\section{RESULTS}

Seventy-one patients who underwent posterior repair augmented with perforated porcine dermal grafts were reviewed. Vaginal incision healing properties were compared to a cohort of 127 previous patients that received posterior repair with nonperforated porcine dermal grafts. The average age of perforated and non-perforated graft patients was similar at 67 years (range 41- 82, median 65) and 62 years (range 28 - 89, median 61 years). Gravidity and parity were similar in both groups as well (perforated: gravidity mean $=3$, median $=3.2$, range $=0-7$, parity mean $=2.8$, median $=2.8$, range $=0-6$; non-perforated: gravidity mean $=3$, median $=3$, range $=0-10$, parity mean $=2.8$, median $=2$, and range $=0-7$ ). There was also no significant difference between the groups when we compared the procedures performed in addition to posterior repair (Table-1).

There was no significant difference between the perforated and non-perforated porcine dermal grafts with respect to tensile strength, suture pull out strength, and flexibility/stiffness (Table-2).

The clinical outcomes are illustrated in Table-3. Of the 127 patients that received a posterior repair with non-perforated grafts, $21(17 \%)$ developed vaginal incision dehiscence compared to 5 of 71 (7\%) patients in the perforated graft group $(\mathrm{p}=0.08)$. All patients with incisional dehiscences were treated conservatively with vaginal estrogen cream initially. All of the patients with perforated grafts and 17/21 patients with the non-perforated grafts healed spontaneously within 4 weeks of instituting vaginal estrogen cream. Four of the patients with non- 
Table 1 - Additional procedures performed with posterior repairs.

\begin{tabular}{lccc}
\hline Procedures & $\begin{array}{c}\text { Perforated Graft } \\
(\mathrm{n}=71)\end{array}$ & $\begin{array}{c}\text { Nonperforated Graft } \\
(\mathrm{n}=127)\end{array}$ & p Value \\
\hline TVT sling & $32(45 \%)$ & $49(39.5 \%)$ & $\mathrm{NS}$ \\
Uterosacral vaginal vault suspension & $13(18.3 \%)$ & $29(22.8 \%)$ & NS \\
LAVH + Uterosacral vaginal vault suspension & $8(11.3 \%)$ & $18(14.1 \%)$ & NS \\
Paravaginal repairs & $28(39 \%)$ & $44(34.6 \%)$ & NS \\
Anterior Repairs & $8(11.3 \%)$ & $20(15.7 \%)$ & NS \\
\hline
\end{tabular}

LAVH = laparoscopically assisted vaginal hysterectomy.

Table 2 - Results of tensile strength, pull out strength, and flexibility testing of perforated and nonperforated porcine dermal grafts.

\begin{tabular}{lccc}
\hline Laboratory Testing & Nonperforated(SD) & Perforated (SD) & p Value* \\
\hline Tensile strengths - pounds & $32.2(23)$ & $34.7(20.7)$ & 0.08 \\
Suture pull out strength - pounds & $7.2(2.7)$ & $6.1(1.8)$ & 0.29 \\
Flexibility/stiffness milligrams & $27.9(2.8)$ & $24.4(12.1)$ & 0.20 \\
\hline
\end{tabular}

Fisher exact test.

Table 3 - Postoperative vaginal incision outcomes.

\begin{tabular}{ll}
\hline Nonperforated $(\mathrm{n}=127)$ & $21(16.5 \%)$ \\
Perforated $(\mathrm{n}=71)$ & $5(7.0 \%)$ \\
$\mathrm{p}$ Value & 0.08 \\
\hline
\end{tabular}

Fisher exact test.

perforated grafts required surgical revision to facilitate healing because of persistent graft exposure after 4 weeks. All of the incisional dehiscences requiring surgical revision were greater than $2 \mathrm{~cm}$ (range 2-4 $\mathrm{cm}$ ) while those that healed spontaneously were less than $2 \mathrm{~cm}$. There were no graft expulsions in either group.

\section{COMMENTS}

We began augmenting our pelvic floor repairs in 1998 with human cadaveric dermal allografts and have reported on its use for augmenting rectocele repairs, vaginal evisceration repair, levatorplasty release and reconstruction as well as rectovaginal fistula (9-12). Because of the advantages it offers over human dermis, such as abundant supply, easy storage (does not require refrigeration), easy handling, good tensile strength, and decreased risk of bacterial or viral transmission, we began using Pelvicol ${ }^{\mathrm{TM}}$ (C.R. Bard, Covington, GA) to augment pelvic floor repairs. While we were satisfied with the clinical results using Pelvicol ${ }^{\mathrm{TM}}$, restoration of the vagina and correction of the rectocele, we began noticing that a number of patients were experiencing immediate postoperative incisional wound dehiscenses. To facilitate faster fibroblast migration and neovascularization, both of which improve healing, we began perforating the porcine grafts. Because we universally trim the grafts to fit the dimensions of the vagina we did not feel that the perforations significantly altered the graft integrity. This was confirmed in the laboratory tensile testing of the perforated and non-perforated grafts.

In the current study, we found that patients who received perforated porcine dermal grafts had fewer problems with incisional healing than did patients with non-perforated grafts. While neither group experienced any severe complications of infection or graft expulsion, four patients in the non-perforated graft 
group required surgical revision. This primarily involved a small vaginal mucosal excision to revise the skin edges and closure with a delayed absorbable suture.

Clinically, we have seen improved healing and decreased vaginal incisional dehiscence since we began perforating the porcine dermal grafts. The vaginal incision dehiscences that have occurred in the perforated graft patients have been small and associated with less inflammation than the dehiscences we encountered with non-perforated grafts. While graft perforation did not reduce wound dehiscences to zero in the perforated group, when dehiscence did occur they responded rapidly to conservative treatment. Recently a manufactured porcine dermal graft with perforation (Pelvisoft ${ }^{\mathrm{TM}}$ Biomesh, C.R. Bard, Cranston, RI) was introduced to the market and was found to have favorable results in a small series of patients (13).

As so often stated, "the search for the ideal graft material is ongoing". We acknowledge that data supporting the wide spread use of interpositional grafts in reconstructive pelvic surgery is lacking. Even though we only evaluated the impact of porcine dermal graft perforation on vaginal incision healing, we feel the results of this study, while retrospective, are encouraging. We enthusiastically await the results of the ongoing trials involving the placement of interpositional grafts and prosthesis in the vagina to augment repairs for pelvic organ prolapse.

\section{CONFLICT OF INTEREST}

None declared.

\section{REFERENCES}

1. Abdel-Fattah M, Barrington JW, Arunkalaivanan AS: Pelvicol pubovaginal sling versus tension-free vaginal tape for treatment of urodynamic stress incontinence: a prospective randomized three-year follow-up study. Eur Urol. 2004; 46: 629-35.

2. Leboeuf L, Miles RA, Kim SS, Gousse AE: Grade 4 cystocele repair using four-defect repair and porcine xenograft acellular matrix (Pelvicol): outcome measures using SEAPI. Urology. 2004; 64: 282-6.
3. Graul ES, Hurst B. Porcine allograft in the repair of anterior and posterior vaginal defects. Urogynecol $\mathbf{J}$ Pelvic Floor Dysfunct. 2002; 13 (Suppl 1): S36.

4. Gomelsky A, Rudy DC, Dmochowski RR.Porcine dermis interposition graft for repair of high grade anterior compartment defects with or without concomitant pelvic organ prolapse procedures. J Urol. 2004; 171: 1581-4.

5. Moore RD, Miklos JR, Kohli N: Posterior repair with Pelvicol dermal graft augmentation. Int Urogynecol J Pelvic Floor Dysfunction. 2003; 14 (Suppl 1): S2.

6. LinksCole E, Gomelsky A, Dmochowski RR: Encapsulation of a porcine dermis pubovaginal sling. J Urol. 2003; 170: 1950.

7. Abramov Y, Gandhi S, Goldberg RP, Botros SM, Kwon C, Sand PK: Site-specific rectocele repair compared with standard posterior colporrhaphy. Obstet Gynecol. 2005; 105:314-8.

8. Gandhi S, Kubba LM, Abramov Y, Botros SM, Goldberg RP, Victor TA, et al.: Histopathologic changes of porcine dermis xenografts for transvaginal suburethral slings. Am J Obstet Gynecol. 2005; 192: 1643-8.

9. Kohli N, Miklos JR: Dermal graft-augmented rectocele repair. Int Urogynecol J Pelvic Floor Dysfunct. 2003; 14: 146-9.

10. Moore RD, Miklos JR: Repair of a vaginal evisceration following colpocleisis utilizing an allogenic dermal graft. Int Urogynecol J Pelvic Floor Dysfunct. 2001; 12:215-7.

11. Miklos JR, Kohli N, Moore R: Levatorplasty release and reconstruction of rectovaginal septum using allogenic dermal graft. Int Urogynecol J Pelvic Floor Dysfunct. 2002; 13: 44-6.

12. Moore RD, Miklos JR, Kohli N: Rectovaginal fistula repair using a porcine dermal graft. Obstet Gynecol. 2004; 104: 1165-7.

13. Dell JR, O'Kelley KR: PelviSoft BioMesh augmentation of rectocele repair: the initial clinical experience in 35 patients. Int Urogynecol J Pelvic Floor Dysfunct. 2005; 16: 44-7; discussion 47.

Accepted after revision:

November 12, 2007

\footnotetext{
Correspondence address:

Dr. G. Bernard Taylor

Department of Obstetrics and Gynecology

Carolinas Medical Center, P.O. Box 32861

Charlotte, North Carolina, 28232-2861, USA

Fax: + 1704355-1941

E-mail: Bernard.taylor@ carolinashealthcare.org
} 


\section{EDITORIAL COMMENT}

During the last decades, different surgical procedures have been introduced to treat urinary incontinence and vaginal prolapse, especially the use of mesh has been claimed to be more effective because conventional repairs may have a high longterm recurrence rate. However, looking into the literature, only few randomized clinical trials are presented regarding the efficacy and recurrence rate of a conventional anterior repair. The reason for this is unclear. Recently, I discussed how to perform a conventional anterior repair with my colleagues. All are well skilled surgeons, but after a short discussion it appeared that all performed a "standard anterior vaginal repair" differently. The discussion disclosed that both different surgical dissection and different suturing material and techniques were used. I think, this observation is universal, i.e. a standard anterior repair are performed very differently, depending on the surgeons own experience and the attitude in each country to develop standards for vaginal reconstructive surgery.

Another reason for the high recurrence rate of conventional vaginal reconstructive surgery could be due to insufficiently knowledge of pelvic anatomy to define the best surgical procedures. We also need to understand how our surgical procedures interfere with pelvic function, which includes the interference with bladder and bowel function. Thus, a patient with prolapse of the anterior vaginal wall will be offered the same surgical procedure, but most often a women with cystocele have different complains. You may see one patient who has no lower urinary tract symptoms, whereas another one has severe urinary tract dysfunction associated with her cystocele. Should they have the same repair?

According to the above-mentioned difficulties, we still need further information on efficacy and long-term results of conventional vaginal reconstructive surgery. Despite of this, new vaginal surgical procedures are introduces rapidly, which frequently imply a high degree of knowledge regarding pelvic anatomy and surgical principles. Many of the procedures demand extensive vaginal dissection and in some instances the surgeon has to operate in anatomical compartments of which the surgeon has no previous experience. The latter is especially a problem regarding procedures including perforation of the obturator foramen, procedures that interferes with bowel function and procedures that imply anchoring of the mesh "in tissue" close to the spine. The latter is in most instances the arcus tendineous of the levator ani muscle, but is the surgeons always aware of the position of this structure, and that it sometimes are detached from the spine due the previous vaginal deliveries? On the other hand, some of the new techniques have proved its superiority compared to previous techniques, as they can be done more quickly, less invasively and more safely. Furthermore, many of the procedures can be performed in the outpatient clinic and with lesser inference with the patients' daily function. Some of the procedures have proven a very high cure rate such as the TVT-procedures.

Another problem is the presence of complications not observed previously. When new surgical procedures are introduced without sufficient knowledge on the efficacy and safety, the patient may receive a treatment, which can be associated with severe complications or an insufficient cure rate. Both factors are most often not observed during the introduction period of a new surgical procedure, but appear obviously later. One example is the complication observed after bone anchors. Another is the presence of mesh erosion through the vaginal mucosa and bowel, bladder and urethral perforations. Furthermore, severe infections after TVT-O procedures have been observed. Dyspareunia due to shrinkage of the vaginal mucosa has been observed as well.

These complications have proven to be a significant challenge. Most often because the mesh material is incorporated into fibrous tissue and in some patients only a part of the mesh can be removed. Frequently, we do not know the right surgical procedures in such cases. Furthermore, what to do in cases with recurrence after insertion of a synthetic mesh? 
In conclusion, I think we need further information on the long-term results following conventional vaginal repairs. I do recognize, that new procedures for vaginal repair are needed, obviously in patients with recurrence. However, these procedures should be evaluated carefully, especially when a new procedure implies a first line treatment, i.e. when the treatment is not limited to patients with recurrence.

Dr. Martin Rudnicki

Associate Professor Department of Obstetrics and Gynecology Roskilde University Hospital Roskilde, Denmark 